\title{
New Approach to the Dark Energy Problem Solution and Consequences
}

\author{
Branko M. Novakovic
}

\begin{abstract}
The consequences of non - vacuum solution of the field equations in derivation of the universe motion model are discussed. Thus, the inclusion of the gravitational energy momentum tensor EMT and the exclusion of the cosmological constant $\Lambda$ in field equations generates both repulsive and attractive gravitational forces. The repulsive gravitational force has a role of the dark energy source. Regularity condition of the related line element gives the limitations to the gravitational radius $r$ that is greater than $G M / 2 c^{2}$ and less than infinity. This means that presented solution has no singularity at the minimal radius. The minimal diameter $L_{\min }$ of the universe mass $M$ is equal to $2 r_{\text {min }}=G M / c^{2}$ that corresponds to the Planck's length $L_{p l}=G M_{p l} / c^{2}$, as the minimal diameter of the Planck's mass $M_{p l}$. From the universe velocity equation, we obtain the limitations to the energy conservation constant $\boldsymbol{\kappa}$ that is greater than zero and less or equal to one. This means that the kinetic energy is less or equal to the potential energy. For that case the spatial curvature of the space $\mathcal{K}$ is greater or equal to zero. In other words, our universe is a flat or a hyper-spherical, because hyperbolic universe is excluded. Further, the zero points of the universe velocity equation determine the minimal gravitational radius at $r_{\min }=G M /(1+\kappa) c^{2}$ and maximal gravitational radius at $r_{\text {max }}=G M /(1-\kappa) c^{2}$. Applying radial density $\rho_{r}=M / r$ to the minimal and maximal gravitational radiuses we obtain the other limitations to the energy conservation constant $\kappa$ that is greater than zero and less than one. For that case the spatial curvature of the space $\mathcal{K}$ is greater than zero. This means that our universe is a hyper-spherical because flat and hyperbolic universes are excluded by the mentioned limitations.
\end{abstract}

Index Terms- Dark Energy problem; Repulsive gravitational force; Minimal gravitational radius; Maximal gravitational radius

\section{INTRODUCTION}

As it is well known, there exist more dynamic models of the universe motion. The most known is the model that has been developed independently by Alexander Friedmann [1], Georges Lemaitre [2], Howard Percy Robertson [3-5] and Arthur Geoffrey Walker [6]. Therefore, it has been named Friedmann - Lemaitre - Robertson - Walker (FLRW) model. The FLRW model describes a homogeneous, isotropic expanding or contracting universe. The general metric has been introduced on the assumption that the universe geometric properties are homogeneous and isotropic, i.e. the Cosmological Principle is valued. Empirically, this is justified on scales larger than $\sim 100 \mathrm{Mpc}$. They also used the Einstein field equations for derivation of the "scale factor" of the universe as a function of time. The FLRW model is well described in the references [1-7]. The history and present state of the universe motion models have been presented in [1-24]. Recently, a new method for testing of the

Branko Novakovic, FSB - University of Zagreb, Luciceva 5, P.O.B. 509, 10000 Zagreb, Croatia
Cosmological Principle by using an isotropic blackbody cosmic microwave background radiation, as evidence for a homogeneous universe, has been presented in [17].

Today, we know that the universe continues to expand, even at an accelerating rate. The main reason for it is an unknown energy called a dark energy. This energy occupies about 68 percent of the total energy in the universe. Therefore, it is crucially important to found out the physical source of the dark energy. One of the candidates for the source of dark energy is a cosmological constant $\Lambda$. Further, the all models of the universe motion should answer to the important questions of the cosmology: what occurred at the initial singularity?, how old is the universe?, how big is the universe?, and what is its ultimate fate?

Recently, a new approach to the solution of the field equations in a gravitational field is presented in the Relativistic Alpha Field Theory (RAFT) [25-27]. This approach is based on the non-vacuum solution of the field equations by including gravitational energy - momentum tensor EMT and excluding cosmological constant $\Lambda$. The physical properties of the gravitational EMT are presented in [28]. Following the references [25-28], the process of derivation of the new universe motion model has been presented in detail in the reference [29]. In the same reference, the new model is compared with the well-known FLRW model. Further, in [29] it has been theoretically proved that the positive (repulsive) gravitational force could be the source of the dark energy.

In this paper we continue with analysis of the properties of the new universe motion model derived in [29]. In that sense, the consequences of the new dynamic model of the universe motion, based on the non-vacuum solution of the field equations are presented. Thus, this model generates both repulsive and attractive gravitational forces. The repulsive force is produced in the region $G M / 2 c^{2} \leq r<G M / c^{2}$. Here, as usually, $G$ is gravitational constant, $M$ is total gravitational mass in universe, $c$ is a speed of the light in vacuum and $r$ is a gravitational radius. At the radius $r=G M / 2 c^{2}$ repulsive gravitational force is maximal and at the radius $r=G M / c^{2}$ is equal to zero. Further, in the region $G M / c^{2}<r<\infty$ the gravitational force is attractive as we know from the experience. At the radius $r=G M / c^{2}$ gravitational force is changing from repulsive to the attractive one (in the expanding phase) and from attractive to repulsive force (in the contracting phase). From the observations we know that our universe is expanding in the acceleration rate. Thus, we can conclude that the present universe radius should be less than $G M / c^{2}$. From the previous consideration we can see that the repulsive gravitational energy has the role of dark energy. In that sense, one can conclude that the dark energy problem is solved. From the structure of the presented model we also can see that the gravitational energy - momentum tensor EMT 
has an effect as the feedback in space-time in order to control of the universe motion.

Further, the regularity condition of the related line element gives the limitations to the energy conservation constant $0<\kappa$ $\leq 1$. This means that the kinetic energy is less or equal to the potential energy. For that case the spatial curvature of the space is greater or equal to zero $\mathcal{K} \geq 0$. In other words, our universe is a flat or a hyper-spherical, because hyperbolic universe is excluded. The zero points of the universe velocity equation determine the minimal gravitational radius at $r_{\min }=$ $G M /(1+\kappa) c^{2}$ and maximal gravitational radius at $r_{\max }=$ $G M /(1-\kappa) c^{2}$. Applying radial density $\rho_{r}=M / r$ to the minimal and maximal gravitational radiuses we obtain the other limitations to the energy conservation constant $0<\kappa<1$ and to the spatial curvature of the space $\mathcal{K}>0$. In this case our universe is a hyper-spherical because flat and hyperbolic universes are excluded by the limitations of the energy conservation constant $\kappa$ and the spatial curvature of the space $\mathcal{K}$. One of the important consequences of the non-vacuum solution of the field equations [25-27], by including gravitational energy - momentum tensor EMT and excluding cosmological constant $\Lambda$, is the existence of the minimal gravitational radius at $r=G M / 2 c^{2}$. This is very important information, because it tells us that the gravitational field has no singularity point at the extremely strong gravitational field.

\section{BRIEF COMPARISON OF THE FREDMAN EQUATIONS AND PRESENTED UNIVERSE MOTION MODEL}

Following the assumption that the universe geometric properties are homogeneous and isotropic, i.e. the Cosmological Principle is valid, Friedman has been derived his equations [1]:

$$
\begin{aligned}
& \left(\frac{\dot{\alpha}}{\alpha}\right)^{2}+\frac{\mathcal{K} c^{2}}{\alpha^{2}}-\frac{\Lambda c^{2}}{3}=\frac{8 \pi G}{3} \rho, \\
& \frac{\ddot{\alpha}}{\alpha}=-\frac{4 \pi G}{3}\left(\rho+\frac{3 p}{c^{2}}\right)+\frac{\Lambda c^{2}}{3} .
\end{aligned}
$$

Here $\alpha(t)$ is the scale factor with related time derivations $\dot{\alpha}$ and $\ddot{\alpha}, \mathcal{K}$ is spatial curvature parameter, $\Lambda$ is cosmological constant, while $\rho$ and $p$ are fluid mass density and pressure, respectively. The presented equations (1) are the basis of the standard big bang cosmological model including the current $\Lambda \mathrm{CDM}$ model. Following the mentioned assumption that the universe is isotropic and homogenous, the model (1) can be used as a first approximation for the evolution of the real, lumpy universe, because it is simple for calculation. Furthermore, the models which calculate the lumpiness in the universe can be added to this model as an extension.

The second equation in (1) states that both the energy density and the pressure cause the expansion rate of the universe $(\ddot{\alpha})$ to decrease. It means that both the energy density and the pressure cause a deceleration in the expansion of the universe. This is the consequence of gravitation, including that pressure is playing a similar role to that of energy (or mass) density. This is, of course, in accordance with the principles of general relativity. On the other side, the cosmological constant, $\Lambda$, causes acceleration in the expansion of the universe.

Friedmann also used the full form of the Einstein's field equations in the General Theory of Relativity that is given by the relation

$$
R_{\mu \eta}-\frac{1}{2} g_{\mu \eta} R+\Lambda g_{\mu \eta}=\frac{8 \pi G}{c^{4}} T_{\mu \eta}, \mu, \eta=0,1,2,3 .
$$

In this relation $R_{\mu \eta}$ is Ricci tensor, $g_{\mu \eta}$ is metric tensor, $R$ is Ricci scalar, $\Lambda$ is the Einstein's cosmological constant, $G$ is the Newton's gravitational constant, $c$ is the speed of light in a vacuum and $T_{\mu \eta}$ is the energy-momentum tensor. Determination of the time evolution of the scale factor $\alpha(t)$ requires the Einstein's field equations together with a way of calculation of density, $\rho(t)$, such as a cosmological equation of state. If the energy-momentum tensor, $T_{\mu \eta}$, is similarly assumed to be isotropic and homogenous, then we have an analytic solution to the Einstein's field equations (2), giving the Friedman equations in the form (1).

The new approach to derivation of the universe motion model is based on the new Relativistic Alpha Field Theory (RAFT) presented in [25-27]. The RAF theory extended the application of the standard GRT to the extremally strong gravitational field with inclusion of the Planck's scale. In this theory the non-vacuum solution of the field equations (2), with inclusion of the gravitational energy - momentum tensor $T_{\mu \eta}$ and without cosmological constant $\Lambda$, is presented. On that way we obtain the solution of the field parameters in the extremally strong gravitational field. In the case of vacuum, the mentioned field parameters are reduced to the well known Schwarzschild vacuum solution of the field equations.

In the process of derivation of the equations of the universe motion we used the related Lagrangean, based on the solution of the line element in RAF theory. The general nondiagonal form of the line element, $\mathrm{ds}^{2}$, in the spherical polar coordinates, can be described by the equation [25]:

$$
d s^{2}=-v c^{2} d t^{2}+2 \lambda c d t d r+d r^{2}+r^{2} d \theta^{2}+r^{2} \sin ^{2} \theta d \phi^{2} .
$$

Here $v$ and $\lambda$ are field parameters, $c$ is the speed of light in a vacuum, $r$ is a radius vector, $\theta$ is an angle between radius vector $r$ and $z$-axis, and $\phi$ is an angle between projection of a radius vector $r$ on $(x-y)$ plane and $x$-axis. Applying field equations

$$
R_{\mu \eta}-\frac{1}{2} g_{\mu \eta} R=\frac{8 \pi G}{c^{4}} T_{\mu \eta}, \quad \mu, \eta=0,1,2,3,
$$

we obtain the solutions of the field parameters $v$ and $\lambda[25]$ :

$$
\begin{aligned}
T_{\mu \eta} \neq 0, \quad \Lambda & =0, \rightarrow v=1-\frac{2 G M}{r c^{2}}+\left(\frac{G M}{r c^{2}}\right)^{2}, \\
\lambda & =\sqrt{\frac{2 G M}{r c^{2}}-\left(\frac{G M}{r c^{2}}\right)^{2}} .
\end{aligned}
$$

Here $G M / c^{2}$ is the Newton's constant of integration, $G$ is a gravitational constant, $M$ is a total gravitational mass, $r$ is a gravitational radius and $c$ is the speed of light in a vacuum. If 
displacement four-vector $d X$ is defined in the frame $K$ by the expression:

$$
d X \rightarrow K(c d t, d r, d \theta, d \phi)=\left\{d x^{i}\right\}, \quad i=0,1,2,3,
$$

then the related covariant metric tensor of the line element (3) has the following matrix presentation [25]

$$
\left[g_{\mu \eta}\right]=\left[\begin{array}{cccc}
-v & \lambda & 0 & 0 \\
\lambda & 1 & 0 & 0 \\
0 & 0 & r^{2} & 0 \\
0 & 0 & 0 & r^{2} \sin ^{2} \theta
\end{array}\right]
$$

Here the non-null components of the metric tensor $g_{\mu \eta}$ are given by the relations [25]:

$$
\begin{gathered}
g_{00}=-v=-\left(1-\frac{2 G M}{r c^{2}}+\left(\frac{G M}{r c^{2}}\right)^{2}\right), \quad g_{11}=1, g_{22}=r^{2}, \\
g_{01,10}=\lambda=\sqrt{\frac{2 G M}{r c^{2}}-\left(\frac{G M}{r c^{2}}\right)^{2}}, \quad g_{33}=r^{2} \sin ^{2} \theta .
\end{gathered}
$$

The related determinant of the metric tensor (7) has the form [25]:

$$
\begin{gathered}
\operatorname{det}\left[g_{\mu \eta}\right]=-r^{4}\left(v+\lambda^{2}\right) \sin ^{2} \theta, \quad r=1, \theta=\frac{\pi}{2}, \rightarrow \\
\operatorname{det}\left[g_{\mu \eta}\right]=-\left(v+\lambda^{2}\right)=-1, \quad v=1-\lambda^{2} .
\end{gathered}
$$

In the previous relation we use the normalization for $r=1$, and $\theta=\pi / 2$. On that way, the well-known condition for the metric tensor of the line element, $\operatorname{det}\left(g_{\mu \eta}\right)=-1$, is satisfied. As the result we obtain the simple relation between field parameters $v$ and $\lambda$.

By including metric parameters (8) and (9) into the field equations (4), we obtain the related gravitational energy momentum tensor (EMT) [28]

$$
\left[T_{\mu \eta}\right]=\left[\begin{array}{rrcc}
v & -\lambda & 0 & 0 \\
-\lambda & -1 & 0 & 0 \\
0 & 0 & r^{2} & 0 \\
0 & 0 & 0 & r^{2} \sin ^{2} \theta
\end{array}\right] \frac{(G M)^{2}}{8 \pi G r^{4}} .
$$

It is easy to prove that this EMT satisfies the required properties [28]: 1) symmetry, $T_{\mu \eta}=T_{\eta \mu}$;2) non-negative energy density for static and free field, $T_{00} \geq 0$; and 3 ) zero trace, $T=0$.

Using the related Lagrangean, based on the solution of the line element in a gravitational field we obtain the first form of the universe motion model as function of the gravitational mass $M$ and radius $r$ that has been derived in the reference [29]:

$$
\begin{gathered}
\dot{r}= \pm\left[\frac{2 G M}{r}\left(1-\frac{G M}{2 r c^{2}}\right)+\left(\kappa^{2}-\varepsilon^{2}\right) c^{2}\right]^{1 / 2}, \\
\ddot{r}=-\frac{G M}{r^{2}}\left(1-\frac{G M}{r c^{2}}\right) .
\end{gathered}
$$

Here $\kappa$ is the energy conservation constant and $\varepsilon$ is the related Lagrangean parameter. Now applying a mass density $\rho$ and pressure $p$ for an adiabatic process, as well as parameter $\varepsilon=1$ (for time-like geodesics) and spatial curvature constant $\mathcal{K}=$ $\left(1-\kappa^{2}\right)$, the model $(11)$ is transformed into the new dynamic model of the universe motion as function of the mass density $\rho$ and pressure $p$ and gravitational radius $r$ [29]:

$$
\begin{aligned}
& \left(\frac{\dot{r}}{r}\right)^{2}+\frac{\mathcal{K} c^{2}}{r^{2}}=\frac{8 \pi G}{3} \rho\left(1-\frac{2 \pi G}{3} \frac{\rho r^{2}}{c^{2}}\right), \\
& \frac{\ddot{r}}{r}=-\frac{4 \pi G}{3}\left(\rho+\frac{3 p}{c^{2}}\right)\left(1-\frac{4 \pi G}{3} \frac{\rho r^{2}}{c^{2}}\right) .
\end{aligned}
$$

This model is valid for $T_{\mu \eta} \neq 0$ and $\Lambda=0$.

Comparing the equations (12) with the Friedman equations (1), we can conclude that the gravitational radius $r$ in (12) has the roll of the scale factor $\alpha$ in (1). The first part on the right side of the second equation in (12) states that both the energy density and the pressure cause a deceleration in the expansion of the universe. This is the consequence of gravitation, including that pressure is playing a similar role to that of energy (or mass) density. This is, of course, in accordance with the principles of general relativity.

On the other hand, the second part of the right side of the second equation in (12) causes acceleration in the expansion of the universe if $4 \pi G \rho r^{2} / 3 c^{2}>1$ and the deceleration if $4 \pi G \rho r^{2} / 3 c^{2}<1$. In the case that $4 \pi G \rho r^{2} / 3 c^{2}=1$ the acceleration of the universe motion is equal to zero. At this point the acceleration is changed into the deceleration (in the expansion phase) and the deceleration is changed into the acceleration (in the contraction phase). This is the consequence of the non-vacuum solution of the field equations (4) with inclusion of the gravitational energy momentum tensor $T_{\mu \eta}(10)$ and exclusion of the cosmological constant $\Lambda$. Thus, we can see that the model of the universe motion (12) generates both repulsive and attractive gravitational forces. In that sense the problem of the dark energy is solved, because the repulsive gravitational energy has the role of the dark energy. Of course, this should be confirmed by the related experiments.

Finally, the main differences between Friedman model (1) and the new non-vacuum solution model (12) are as follows:

a) in the Friedman model the expansion of the universe motion is caused by the Dark Energy that is presented by the cosmological constant $\Lambda$. But we do not know what is the physical source of the Dark Energy,

b) on the contrary, in the new model of the universe motion (12), which is based on the non-vacuum solution of the field equations, the source of the both expansion and contraction is the gravitational field. 


\section{III.CONSSEQUENCES OF THE NEW UNIVERSE MOTION MODEL}

\section{A. Regularity of the metrics of the line element}

The line element (3) with parameters (5) can be rewritten into the following form

$$
\begin{gathered}
d s^{2}=-\left(1-\frac{G M}{r c^{2}}\right)^{2} c^{2} d t^{2}+2 \sqrt{\frac{2 G M}{r c^{2}}\left(1-\frac{G M}{2 r c^{2}}\right)} c d t d r \\
+d r^{2}+r^{2} d \theta^{2}+r^{2} \sin ^{2} \theta d \phi^{2}
\end{gathered}
$$

It is easy to prove that the line element (13) is regular if the following conditions are satisfied:

$$
\left(1-\frac{G M}{2 r c^{2}}\right) \geq 0 \rightarrow \frac{G M}{2 r c^{2}} \leq 1 \rightarrow \frac{G M}{2 c^{2}} \leq r<\infty .
$$

Thus, the region of the regularity of the line element (13) is determined by the last relation in (14). Further, from the previous relations we can conclude that there exists a minimal gravitational radius $r_{\min }$ which still preserves regularity of the line element (13)

$$
r_{\min }=\frac{G M}{2 c^{2}}
$$

Including of the minimal gravitational radius (15) into the line element (13) we obtain regular line element in the form

$$
d s^{2}=-c^{2} d t^{2}+d r^{2}+r^{2} d \theta^{2}+r^{2} \sin ^{2} \theta d \phi^{2} .
$$

For the radiuses less than minimal gravitational radius $r_{\text {min }}$ the line element (13) becomes imaginary item. Gravitational radius can be equal to zero only if the mass is equal to zero. This means that any mass $m \neq 0$ can not reach the singularity at $r=0$ in a gravitational field. The minimal gravitational radius is four time less than Schwarzschild radius

$$
r_{s}=\frac{2 G M}{c^{2}}=4 r_{\min }=4 \frac{G M}{2 c^{2}}
$$

\section{B. The physical meaning of the minimal gravitational radius}

In order to highlight the physical meaning of the minimal gravitational radius $r_{\text {min }}$, one can apply of the Planck's mass $M_{p}$ to the relation (15)

$$
r_{p \min }=\frac{G M_{p}}{2 c^{2}}
$$

Here $r_{p \min }$ is a minimal radius of the Planck' mass $M_{p}$. As it is well-known, the Planck's length $L_{p}$ [30] and the Planck's mass $M_{p}$ [31] are defined from three fundamental physical constants: the speed of light in vacuum $c$, the reduced Planck's constant $\hbar$ and the gravitational constant $G$ :

$$
M_{p}=\sqrt{\frac{\hbar c}{G}}, \quad L_{p}=\sqrt{\frac{\hbar G}{c^{3}}} .
$$

Assuming that the Planck's mass $M_{p}$ is spherically symmetric body and following the relations (18) and (19), we can calculate the following equality:

$$
\begin{aligned}
\frac{M_{p}}{2 r_{p \min }} & =\frac{c^{2}}{G}, \quad \frac{M_{p}}{L_{p}}=\frac{\sqrt{\hbar c / G}}{\sqrt{\hbar G / c^{3}}}=\frac{c^{2}}{G}=\text { const } . \\
\rightarrow 2 r_{p \text { min }}=L_{p} \rightarrow r_{p \text { min }} & =\frac{L_{p}}{2} .
\end{aligned}
$$

From the last relation in (20), one can conclude that the minimal gravitational radius of the Planck's mass $M_{p}$, denoted by $r_{p m i n}$, is equal to the half of the Planck's length $L_{p}$. Thus, the physical meaning of the Planck's length could be: $L_{p}$ is the gravitational minimal diameter (length) of the Planck's mass $M_{p}$. On the other words, Planck's Mass $M_{p}$ can not have a gravitational diameter less than Planck's length $L_{p}$. Furthermore, from the relation (14) we can see that the minimal radius is proportional to the gravitational mass. Thus, the smallest minimal radius belongs to the smallest mass in space - time. Since the Planck's mass is not the smallest mass in space - time, the Planks length is not the smallest length in that sense.

From the relation (15) one can derive maximal radial density in gravitational field $\rho_{r_{\max }}$ :

$$
\begin{gathered}
\frac{M}{2 r_{\text {min }}}=\frac{M}{L_{\text {min }}}=\frac{c^{2}}{G}=\text { const. } \rightarrow \\
\rho_{r_{\text {max }}}=\frac{M}{r_{\text {min }}}=\frac{M_{p}}{r_{p_{\text {min }}}}=\frac{2 c^{2}}{G}=\text { const. }
\end{gathered}
$$

Thus, the minimal gravitational radius of mass $M$ corresponds to the minimal gravitational radius $r_{p \min }$ of the Planck's mass $M_{p}$. On the same way, the minimal gravitational length $L_{\min }$ is the minimal diameter of the mass $M$. From relations (21) we can see that the maximal radial density $\rho_{\mathrm{rmax}}$ is at the minimal gravitational radius and is the constant and the same for all masses including Planck's mass.

\section{Universe velocity and acceleration at the characteristic radiuses}

Following of the derivation of the universe motion model (11), we can see that the sum of the kinetic energy $E_{k}$ and potential energy $E_{p}$ of a particle with unit mass is equal to constant:

$$
\begin{gathered}
E_{k}=\frac{1}{2}\left[\dot{r}^{2}\right], E_{p}=-\frac{1}{2}\left(\frac{2 G M}{r c^{2}}-\left(\frac{G M}{r c^{2}}\right)^{2}\right) \varepsilon^{2} c^{2}, \\
E_{k}+E_{p}=\frac{c^{2}}{2}\left(\kappa^{2}-\varepsilon^{2}\right)=\text { const. }
\end{gathered}
$$

Putting that $\varepsilon=1$ (for time-like geodesics), one obtains the following relations between kinetic and potential energies and the energy conservation constant $\kappa$ :

$$
\begin{aligned}
& E_{k}+E_{p}=\frac{c^{2}}{2}\left(\kappa^{2}-1\right),\left|E_{k}\right|=\left|E_{p}\right| \rightarrow \kappa^{2}=1, \\
& \left|E_{k}\right|>\left|E_{p}\right| \rightarrow \kappa^{2}>1,\left|E_{k}\right|<\left|E_{p}\right| \rightarrow 0<\kappa^{2}<1 .
\end{aligned}
$$


From the relations (11) and (23) and assuming that the universe motion follows time-like geodesic $(\varepsilon=1)$, one can derive radial velocity $\dot{r}$ and radial acceleration $\ddot{r}$ of the universe motion as function of the gravitational mass and radius:

$$
\begin{gathered}
\dot{r}= \pm\left[\frac{2 G M}{r}\left(1-\frac{G M}{2 r c^{2}}\right)+\left(\kappa^{2}-1\right) c^{2}\right]^{1 / 2}, \\
\ddot{r}=-\frac{G M}{r^{2}}\left(1-\frac{G M}{r c^{2}}\right) .
\end{gathered}
$$

The sign (+) in the velocity equation is valid for an expanding phase, while the sign (-) is related to the contracting one. The acceleration equation tells us that the universe acceleration becomes repulsive if $\left(G M / r c^{2}\right)>1$. One of the conclusions could be that the repulsive gravitational force is the source of dark energy. For the case $\left(G M / r c^{2}\right)=1$ the acceleration is equal to zero and for $\left(G M / r c^{2}\right)<1$ the acceleration is attractive. At the point $\left(G M / r c^{2}\right)=1$, the repulsive acceleration is changing into the attractive one (for expansion phase) and vice versa (for contraction phase).

The velocity equation in (24) has two zeros at the positions $r_{1}$ and $r_{2}$ :

$$
r_{1}=\frac{G M}{(1+\kappa) c^{2}}, \quad r_{2}=\frac{G M}{(1-\kappa) c^{2}} .
$$

From the relations (23) and (25) one can see that the hyperspherical scenario of the universe motion can be realized only for the case where the energy conservation constant $\kappa$ is satisfying the condition $(0<\kappa<1)$. This means that potential energy is greater than kinetic energy. For that case the velocity equation in (24) has two finite zeros: initial one for $r=r_{1}$, and final one for $r=r_{2}$.

On the other side the hyperbolic scenario of the universe motion can be realized only for the case where the energy conservation constant $\kappa$ is greater than one $(\kappa>1)$. This means that kinetic energy is greater than potential energy. For that case the velocity relation (24) has only one real zero $r_{1}$ in (25), because the other one gives a negative $r_{2}$. The real zero $r_{1}$ is the initial one for this scenario of the universe motion.

Finally, the flat universe can be realized only for the case where the energy conservation constant $\kappa$ is equal to one ( $\kappa$ $=1$ ). This means that kinetic energy is equal to potential energy. For that case the velocity equation in (24) has one finite initial zero for $r=r_{1}$, and one infinite final zero for $r_{2} \rightarrow$ $\infty$. The initial radius $r_{l}$ of the hyperbolic universe $(\kappa>1)$ is less than the initial radius of the flat universe $(\kappa=1)$ and also less than initial radius of the hyperspherical universe $(\kappa<1)$.

The maximal radial velocity $\dot{r}_{\max }$ can be obtained from (24) by applying condition that the radial universe acceleration is equal to zero:

$$
\ddot{r}=0 \rightarrow \quad r=r_{c}=\frac{G M}{c^{2}}, \rightarrow \dot{r}_{\text {max }}= \pm \kappa c
$$

Thus, including the values of the energy conservation constant $\kappa$ into (26) we obtain the following relation

$$
\dot{r}_{\text {max-hyperbolic }}>\dot{r}_{\text {max-flat }}>\dot{r}_{\text {max-hyperspherical } .}
$$

Now, including $r_{1}$ and $r_{2}$ from (25) into the accelerating equation of the universe motion (24), we obtain the related accelerations at the radiuses $r_{1}$ and $r_{2}$ :

$$
\begin{aligned}
& \ddot{r}_{1}=-\frac{G M}{r_{1}^{2}}\left(1-\frac{G M}{r_{1} c^{2}}\right)=\frac{\kappa(1+\kappa)^{2} c^{4}}{G M}, \\
& \ddot{r}_{2}=-\frac{G M}{r_{2}^{2}}\left(1-\frac{G M}{r_{2} c^{2}}\right)=\frac{-\kappa(1-\kappa)^{2} c^{4}}{G M} .
\end{aligned}
$$

The first relation in (28) gives the repulsive initial acceleration $\ddot{r}_{1}$ at the radius $r_{1}$ for all scenarios of the universe motion. On the other side the acceleration $\ddot{r}_{2}$ at radius $r_{2}$ of the universe motion is attractive for hyperspherical and hyperbolic scenarios of the universe motion. Meanwhile, for the flat scenario $(\kappa=1)$ radius $r_{2}$ is going to infinity and the acceleration is going to zero.

From the relations (12) and (25) we can derive the relation between parameters $\kappa$ and $\mathcal{K}$ :

$$
\begin{gathered}
\left(1-\kappa^{2}\right)=\mathcal{K}, \quad \kappa^{2}=1 \rightarrow \mathcal{K}=0 \rightarrow \text { Flat universe } \\
\kappa^{2}<1 \rightarrow \mathcal{K}>0 \rightarrow \text { Hyperspherical universe } \\
\kappa^{2}>1 \rightarrow \mathcal{K}<0 \rightarrow \text { Hyperbolic universe. }
\end{gathered}
$$

Here $\kappa$ is the energy conservation constant derived from the Lagrangean [29] and $\mathcal{K}$ is spatial curvature parameter from the Friedmann equations (1).

\section{Limitations of the energy conservation constant $\boldsymbol{\kappa}$.}

For determination of the scenario of the universe motion given by (29), we have to know the limitations of the energy conservation constant $\kappa$. In that sense, one can start with the radial density relation $\rho_{\mathrm{r}}$ at the minimal and maximal universe radiuses:

$$
\begin{gathered}
\rho_{r}=\frac{M}{r}, \rho_{r_{\text {max }}}=\frac{M}{r_{\text {min }}}=\frac{(1+\kappa) c^{2}}{G}, \\
\rho_{r_{\text {min }}}=\frac{M}{r_{\text {max }}}=\frac{(1-\kappa) c^{2}}{G}, \frac{\rho_{r_{\max }}}{\rho_{r_{\text {min }}}}=\frac{(1+\kappa)}{(1-\kappa)}, \\
\rightarrow \kappa=\frac{\rho_{r_{\text {max }}}-\rho_{r_{\text {min }}}<1 .}{\rho_{r_{\text {max }}}+\rho_{r_{\text {min }}}}<1 .
\end{gathered}
$$

From the previous relations we can see the limitations of the energy conservation constant $0<\kappa<1$ and the limitation of the spatial curvature of the space $\mathcal{K}>0$. For that case our universe is a hyperspherical because flat and hyperbolic universes are excluded by limitations of parameter $\kappa$ or parameter $\mathcal{K}$.

\section{CONCLUSION}

The consequences of the new dynamic model of the universe motion, based on the non-vacuum solution of the field equations are presented. This solution includes the gravitational energy - momentum tensor EMT and excludes cosmological constant $\Lambda$. As the first consequence we obtain that both repulsive and attractive forces are generate by gravitational field. The repulsive gravitational force could be 
the source of the dark energy instead of the cosmological constant $\Lambda$. In that sense the dark energy could be seen as a repulsive gravitational energy. On that way the problem of the source of the dark energy is solved. The second consequence, obtained from the regularity condition of the related line element, shows that presented solution has no singularity point at the minimal radius. From the universe velocity equation and radial density at the minimal and maximal gravitational radiuses, we obtain the third consequence: the limitations to the energy conservation constant $0<\kappa<1$ and to the spatial curvature of the space $\mathcal{K}>0$. In that case our universe is a hyperspherical because flat and hyperbolic universes are excluded by the limitations of parameters $\kappa$ and $\mathcal{K}$.

Funding: This work was supported by the National Scientific Foundation of Republic of Croatia (grant number: 120-1201842-3048).

\section{REFERENCES}

[1] A. Friedmann, Über die Möglichkeit einer Welt mit konstanter negativer Krümmung des Raumes, Z.Phys. 21 (1924) 326-332 (in German). A. Friedman, On the possibility of a world with constant negative curvature of space, General Relativity and Gravitation 31 (1999) 2001-2008.

[2] G. Lemaître, Expansion of the universe, a homogeneous universe of constant mass and increasing radius accounting for the radial velocity of extra galactic nebulae, Monthly Notices of the Royal Astronomical Society 91 (1921) 483-490.

[3] H.P. Robertson, Kinematics and world structure, Astrophysical Journal 82 (1935) 284-301.

[4] H.P. Robertson, Kinematics and world structure II, Astrophysical Journal 83 (1936) 187-201.

[5] H.P. Robertson, Kinematics and world structure III, Astrophysical Journal 83 (1936) 257-271.

[6] A. G. Walker, On Milne's theory of world structure. Proceedings of the London Mathematical Society 242 (1937) 90-127.

[7] M. Lachieze-Rey, J.P. Luminet, Cosmic topology, Physical Reports 254 (1995) 135-214.

[8] S. Perlmutter et al., Nature 391 (1998) 51; Riess A. et al., Astron. J. 116 (1998) 1009.

[9] B.M. Novakovic, D.B. Novakovic, A.B. Novakovic, Cosmological constant problem solution valid for both Planck's and cosmological scales. AIP Conference Proceedings 839 (2006) 144. https://doi.org/10.1063/1.2216624

[10] G.M. Kremer, Cosmological models described by a mixture of der Waals fluid and Dark energy, Phys. Rev. D 68 (2003) 123507.

[11] S.E. Jorás, T.J. Stuchi, Chaos in a closed Friedmann Robertson Walker universe: An imaginary approach, Phys. Rev. D 68 (2003) 123525.

[12] M. Susperregi, Dark energy and Dark matter from an inhomogeneous dilaton, Phys. Rev. D 68 (2003) 123509.

[13] P.G. Castro, M. Douspis, P.G. Ferreira, Scale of homogeneity of the universe from WMAP, Phys. Rev. D 68 (2003) 127301.

[14] P.P. Avelino, C. J.A.P. Martins, C. Santos, E.P.S. Shellard, Topological defects: A problem for cyclic universe, Phys. Rev. D 68 (2003) 123502.

[15] J. Khoury, P.J. Steinhardt, N. Turok, Designing cyclic universe models, Phys. Rev. Lett. 92 (2004) 031302.

[16] J. E. Lidsey, Physical Review D 70 (2004) 041302.

[17] T. Clifton, C. Clarkson, P. Bull, Isotropic blackbody: Cosmic microwave background radiation as evidence for a homogeneous universe, Phys. Rev. Lett. 109 (2012) 051303.

[18] M. Lincoln, A. Wasser, Spontaneous creation of the Universe Ex Nihilo, Physics of the Dark Universe 2 (2013) 195-199. https://doi.org/10.1016/j.dark.2013.11.004

[19] M. Baldi, Dark Energy simulations, Dark Universe 1 (2012) 162-193. https://doi.org/10.1016/j.dark.2012.10.004

[20] M. Kuhlen, M. Vogelsberger, R. Angulo, Numerical simulations of the dark universe: State of the art and the next decade, Dark Universe 1 (2012) 50-93. https://doi.org/10.1016/j.dark.2012.10.002
[21] P. Huang, F. F. Yuan, Uniformly expanding vacuum: A possible interpretation of the dark energy, Physics of the Dark Universe 12 (2016) 45-49

[22] S. Ghaffari, H. Moradpour, Valdir B. Bezerra, J.P. MoraisGraça, I. P. Lobo, Tsallis holographic dark energy in the brane cosmology. https://doi.org/10.1016/j.dark.2018.11.007

[23] R. von Marttens, L. Casarini, D.F. Mota, W. Zimdahl, Cosmological constraints on parametrized interacting dark energy. https://doi.org/10.1016/j.dark.2018.10.007

[24] A. Casalino, M. Rinaldi, L. Sebastiani, S. Vagnozzi, Mimicking dark matter and dark energy in a mimetic model compatible with GW170817,

Physics of the Dark Universe 22 (2018) 108-115. https://doi.org/10.1016/j.dark.2018.10.001

[25] Novakovic, B. M.: Relativistic alpha field theory - Part I: Determination of Field Parameters. International Journal of New Technology and Research (IJNTR) ISSN:2454-4116, 1, 5 (2015) 23-30. https://doi.org/10.31871/IJNTR.1.5.15

[26] Novakovic, B. M.: Relativistic alpha field theory-Part II: Does a Gravitational Field Could be Without Singularity? International Journal of New Technology and Research (IJNTR) (2015). https://doi.org/10.31871/IJNTR.1.5.16

[27] Novakovic, B. M.: Relativistic alpha field theory-Part III: Does Gravitational Force Becomes Positive if $\left(\mathrm{GM} / \mathrm{rc}^{2}\right)>1$ ? International Journal of New Technology and Research (IJNTR) (2015). https://doi.org/10.31871/IJNTR.1.5.17

[28] B. M. Novakovic, Physical Properties of Energy-Momentum Tensor of Gravitational Field in RAF Theory, International Journal of New Technology and Research (IJNTR) ISSN:2454-4116, 4, 11 (2018) 19-25. https://doi.org/10.31871/IJNTR.4.11.10

[29] B. M. Novakovic, Is positive Gravitational Force Source of Dark Energy?, International Journal of New Technology and Research (IJNTR) ISSN:2454-4116, 1, 7 (2015) 6-13. https://doi.org/10.31871/IJNTR.1.7.5

[30] CODATA: Constants, Planck length, US National Institute of Standard and Technology, June (2015). (http://physics.nist.gov/cgi-bin/cuu/Value?plkl)

[31] CODATA Value, Planck mass, US National Institute of Standard and Technology, June (2015) (http://physics.nist.gov/cgi-bin/cuu/Value?plkm)

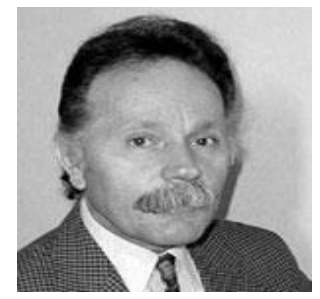

Branko Novakovic is a Professor emeritus at FSB - University of Zagreb, Croatia. Prof. Novakovic received his $\mathrm{PhD}$ from the University of Zagreb in 1978. His research of interest includes physics, control systems, robotics, neural networks, and fuzzy control. He is author of three books; Relativistic Alpha Field Theory (RAFT, e-book, 2016), Control Methods in Robotics, Flexible Manufacturing Systems and Processes (1990), Control Systems (1985) and the first co-author of a book Artificial Neural Networks (1998). He has published over 230 research papers in his research of interest. 